\title{
Suppressing Chaos for a Fractional-Order Chaotic Chemical Reaction Model via PD ${ }^{\zeta}$ Controller
}

\author{
Hui Wang (iD) \\ Department of Finance and Insurance, School of Business Nanjing University, Nanjing 210093, China \\ Correspondence should be addressed to Hui Wang; hwangcn1@sina.com
}

Received 27 November 2021; Revised 19 December 2021; Accepted 10 January 2022; Published 4 February 2022

Academic Editor: Fairouz Tchier

Copyright $\odot 2022$ Hui Wang. This is an open access article distributed under the Creative Commons Attribution License, which permits unrestricted use, distribution, and reproduction in any medium, provided the original work is properly cited.

\begin{abstract}
In this work, based on the earlier publications, we build a new fractional-order chemical reaction model. Computer simulations manifest that the fractional-order chemical reaction model presents chaotic behavior under a certain parameter condition. To eliminate the chaotic dynamical property, a suitable fractional-order $\mathrm{PD}^{\zeta}$ controller with time delay is designed. Regarding the time delay as a bifurcation parameter, we set up a novel delay-independent stability and bifurcation criterion guaranteeing the stability and the creation of Hopf bifurcation of the controlled fractional-order chemical reaction model. The influence of time delay on the stability and Hopf bifurcation of the controlled fractional-order chemical reaction model is revealed. At last, numerical simulations are performed to sustain the rationality of the designed $\mathrm{PD}^{\zeta}$ controller. The obtained conclusions of this work are completely novel and have immense application prospects in the chaos control of chemical reaction systems. Furthermore, the research idea can also be utilized to suppress the chaos of a lot of fractional-order chaotic models.
\end{abstract}

\section{Introduction}

Chaos exists widely in many areas such as climate, physics, chemistry, engineering, economy and finance, complex networks, and population systems [1-6]. The chaotic phenomenon depends on the initial condition of the original system. The chaotic behavior occurring in the nonlinear dynamical systems owns the very complex property and unpredictability. In many cases, chaotic behavior is not what we want in our practical life. Thus, a natural problem arises: how to suppress the chaotic behavior of the original system has been an important theme in many disciplines. During the past several decades, suppressing chaos has received great attention from many scholars. For example, Chen [7] controlled the chaos via a simple adaptive feedback control technique. Du et al. [8] applied the phase space compression method to control the chaos of an economic model. In 1990, Yorke [9] utilized Ott, Grebogi, and Yorke (OGY) control approach to control the chaos of a chaotic model. Paula and Savi [10] designed an extended time-delayed feedback control approach to suppress the chaos of a nonlinear pendulum. For more detailed literature on this aspect, one can refer [11-14].
In 1996, Geysermans and Baras [15] proposed a homogeneous chaotic Wilamowski-Rossler model. The balance equations of this model have a well-defined microscopic counterpart and all the reaction follows the following "elementary" steps:

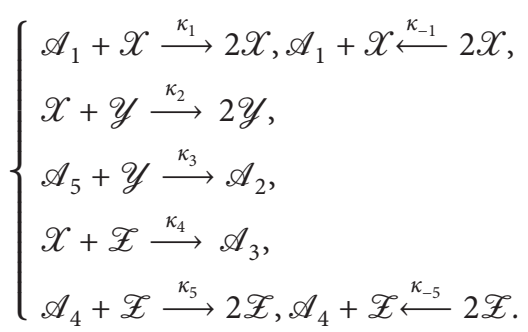

System (1) includes two autocatalytic steps involving constituents $\mathscr{X}$ and $\mathscr{Z}$, coupled via three other steps involving the three constituents $\mathscr{X}, \mathscr{Z}$, and $\mathscr{Y}$. The initial $\left(\mathscr{A}_{1}, \mathscr{A}_{4}, \mathscr{A}_{5}\right)$ and final $\left(\mathscr{A}_{2}, \mathscr{A}_{3}\right)$ product concentrations remain fixed. The distance from thermodynamic equilibrium is controlled by the values of $\mathscr{A}_{1}, \mathscr{A}_{2}, \mathscr{A}_{3}, \mathscr{A}_{4}, \mathscr{A}_{5}$. $\kappa_{ \pm i}(i=1,2,3,4,5)$ stands for the rate constant. In model (1), there are 15 free parameters. To reduce the number of free 
parameters, Geysermans and Baras [15] selected the rate coefficients $\kappa_{-2}=0, \kappa_{-3}=0$, and $\kappa_{-4}=0$. Note that the last two relations imply that $\mathscr{A}_{2}$ and $\mathscr{A}_{3}$ are continuously removed from the reactor $[15,16]$.

Assuming that there exists an ideal mixture and a wellstirred reactor, then the macroscopic rate equations of model (1) can be expressed as follows:

$$
\left\{\begin{array}{l}
\frac{\mathrm{d} u_{1}(t)}{\mathrm{d} t}=\beta_{1} u_{1}(t)-\kappa_{-1} u_{1}^{2}(t)-u_{1}(t) u_{2}(t)-u_{1}(t) u_{3}(t) \\
\frac{\mathrm{d} u_{2}(t)}{\mathrm{d} t}=u_{1}(t) u_{2}(t)-\beta_{5} u_{2}(t) \\
\frac{\mathrm{d} u_{3}(t)}{\mathrm{d} t}=\beta_{4} u_{3}(t)-u_{1}(t) u_{3}(t)-k_{-5} u_{3}^{2}(t)
\end{array}\right.
$$

where $u_{1}(t), u_{2}(t)$, and $u_{3}(t)$ stand for the mole fractions of $\mathscr{X}, \mathscr{Y}$, and $\mathscr{Z}$ at the time $t$. The rate constants $\kappa_{1}, \kappa_{3}$, and $\kappa_{5}$ are incorporated in the parameters $\beta_{1}, \beta_{5}$, and $\beta_{4}$ (e.g., $\left.\beta_{1}=\kappa_{1}\left[\mathscr{A}_{1}\right], \ldots, o\right)$ and $\beta_{1}>0, \beta_{4}>0, \beta_{5}>0, \kappa_{-1}>0, \kappa_{-5}>0$ stand for the constants. In detail, one can refer $[15,16]$. In 2015, Xu and $\mathrm{Wu}$ [17] dealt with the bifurcation control of chaos for model (2) via three-time delay feedback controllers. Namely, they considered the following controller chemical model:

$$
\left\{\begin{array}{l}
\frac{\mathrm{d} u_{1}(t)}{\mathrm{d} t}=\beta_{1} u_{1}(t)-\kappa_{-1} u_{1}^{2}(t)-u_{1}(t) u_{2}(t)-u_{1}(t) u_{3}(t)+\mu_{1}\left[u_{1}(t)-u_{1}(t-\theta)\right] \\
\frac{\mathrm{d} u_{2}(t)}{\mathrm{d} t}=u_{1}(t) u_{2}(t)-\beta_{5} u_{2}(t)+\mu_{2}\left[u_{2}(t)-u_{2}(t-\theta)\right], \\
\frac{\mathrm{d} u_{3}(t)}{\mathrm{d} t}=\beta_{4} u_{3}(t)-u_{1}(t) u_{3}(t)-k_{-5} u_{3}^{2}(t)+\mu_{3}\left[u_{3}(t)-u_{3}(t-\theta)\right],
\end{array}\right.
$$

where $\mu_{i}(i=1,2,3)$ stands for a real constant and $\theta$ is a delay.

It is worth mentioning that all the literature above (see [15-17]) are only concerned with the integer-order chemical models and they are not concerned with the fractional-order chemical models. Recent studies have shown that fractionalorder differential equation is deemed as a more effective tool to portray natural phenomena than the classical integerorder ones since it has great advantages in memory trait and hereditary property of numerous materials and development processes [18-20]. Recently, fractional-order dynamical systems have displayed great application in lots of areas such as network systems, intelligent control, physical science, biological engineering, chemistry, finance, and so on [21-26]. Rich achievements on fractional-order dynamical models have been obtained. For example, Ke [27] dealt with the Mittag-Leffler stability and asymptotic $\omega$-periodicity for a class of fractional-order inertial delayed neural networks. $\mathrm{Du}$ and $\mathrm{Lu}$ [28] focused on the finite-time stability for fractional-order fuzzy delayed cellular neural networks. Xiao et al. [29] probed into the bifurcation control problem for fractional-order small-world networks; Huang et al. [30] studied the bifurcation problem of fractional-order delayed neural networks. In detail, we refer the readers to [31-34].

Inspired by the exploration above and on the basis of system (2), in order to describe the continuous change process of the mole fractions of $\mathscr{X}, \mathscr{Y}$, and $\mathscr{Z}$ and characterize the memory trait and hereditary property of the variables $\mathscr{X}, \mathscr{Y}$, and $\mathscr{Z}$, we modify system (2) as the following fractional-order form:

$$
\left\{\begin{array}{l}
\frac{\mathrm{d}^{\zeta} u_{1}(t)}{\mathrm{d} t^{\zeta}}=\beta_{1} u_{1}(t)-\kappa_{-1} u_{1}^{2}(t)-u_{1}(t) u_{2}(t)-u_{1}(t) u_{3}(t) \\
\frac{\mathrm{d}^{\zeta} u_{2}(t)}{\mathrm{d} t^{\zeta}}=u_{1}(t) u_{2}(t)-\beta_{5} u_{2}(t) \\
\frac{\mathrm{d}^{\zeta} u_{3}(t)}{\mathrm{d} t^{\zeta}}=\beta_{4} u_{3}(t)-u_{1}(t) u_{3}(t)-k_{-5} u_{3}^{2}(t)
\end{array}\right.
$$

where $\zeta \in(0,1]$. The research indicates that when $\zeta=0.97, \beta_{1}=30, \kappa_{-1}=0.55, \beta_{5}=9.5, \beta_{4}=16.5, \kappa_{-5}=0.5$, then there is a chaotic phenomenon in system (3). The software simulation figures are presented in Figure 1.

In this current work, we are to deal with the chaos control of system (4) by virtue of a fractional-order $\mathrm{PD}^{\zeta}$ controller. The key contributions of this study are as follows:

(i) On the basis of the earlier studies, a new fractionalorder chaotic chemical reaction model is set up 


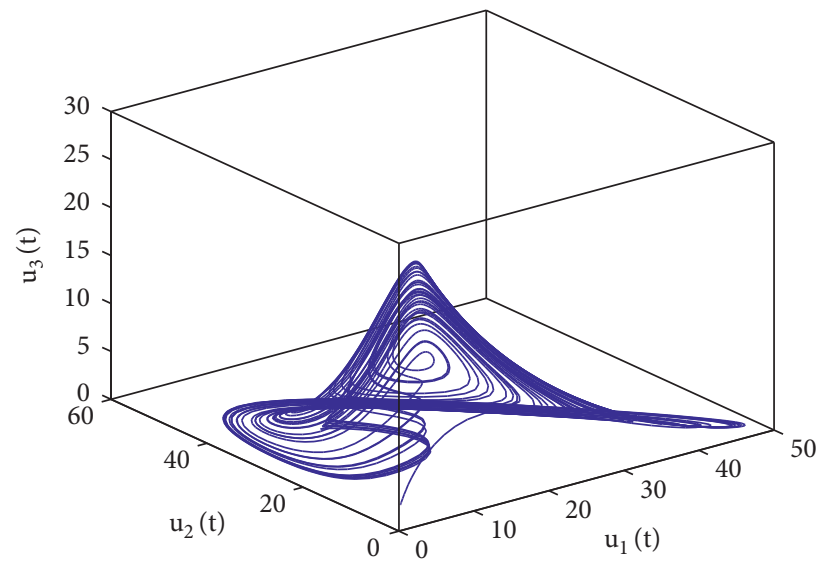

Figure 1: Software simulation figures of system (4) with $\zeta=0.97, \beta_{1}=30, \kappa_{-1}=0.55, \beta_{5}=9.5, \beta_{4}=16.5, \kappa_{-5}=0.5$.

(ii) The chaotic phenomenon of system (4) is suppressed by means of an appropriate fractional-order $\mathrm{PD}^{\zeta}$ controller

(iii) The study approach can be utilized to suppress the chaos of lots of fractional-order dynamical models in many subjects

This manuscript can be arranged as follows: some prerequisite theory on the fractional-order differential equation is prepared in Section 2; in Section 3, we prove the existence and uniqueness of the solution of system (4); in Section 4, the chaos of system (4) is suppressed via fractional-order $\mathrm{PD}^{\zeta}$ controller and a delay-independent sufficient condition that ensures the stability and the creation of Hopf bifurcation of the fractional-order controlled chaotic chemical reaction model is built; in Section 5, software simulation results are presented to sustain the established conclusions; and Section 6 completes this article.

\section{Preliminary Knowledge}

In this part, we present some indispensable theories on a fractional-order differential equation.

Definition 1 (see [35]). The fractional type integral of the order $\zeta$ of the function $u(\xi)$ is given by

$$
\mathscr{I}^{\zeta} u(\xi)=\frac{1}{\Gamma(\zeta)} \int_{\xi_{0}}^{\xi}(\xi-\nu)^{\zeta-1} u(\nu) \mathrm{d} \nu
$$

where $\xi>\xi_{0}, \zeta>0$ and $\Gamma(\nu)=\int_{0}^{\infty} s^{\nu-1} e^{-s} \mathrm{~d} s$.

Definition 2 (see [35]). The Caputo fractional order derivative of the order $\zeta$ of the function $u(\nu) \in\left(\left[\nu_{0}, \infty\right), R\right)$ is defined as follows:

$$
\mathscr{D}^{\zeta} u(\nu)=\frac{1}{\Gamma(\kappa-\zeta)} \int_{\nu_{0}}^{\nu} \frac{u^{(\kappa)}(s)}{(\nu-s)^{\zeta-\kappa+1}} \mathrm{~d} s,
$$

where $\nu \geq v_{0}$ and $\kappa$ represents a positive integer $(\zeta \in[\kappa-1, \kappa))$. In particular, if $\zeta \in(0,1)$, then

$$
\mathscr{D}^{\zeta} u(\nu)=\frac{1}{\Gamma(1-\zeta)} \int_{v_{0}}^{\nu} \frac{u^{\prime}(s)}{(\nu-s)^{\zeta}} \mathrm{d} s .
$$

Lemma 1 (see [36]). Consider the fractional-order system $\mathscr{D}^{\zeta} w=\mathscr{F} w, w(0)=w_{0}$ where $\zeta \in(0,1), w \in R^{l}, \mathscr{F} \in R^{l \times l}$. Assuming that $\chi_{i}(i=1,2, \ldots, l)$ is the root of the characteristic equation of $\mathscr{D}^{\zeta} w=\mathscr{F} w$, then the equilibrium point of the system $\mathscr{D}^{\zeta} w=\mathscr{F} w$ is locally asymptotically stable if $\left|\arg \left(\chi_{i}\right)\right|>(\zeta \pi / 2)(i=1,2, \ldots, l)$ and the equilibrium point of the system $\mathscr{D}^{\zeta} w=\mathscr{F} w$ is stable if $\left|\arg \left(\chi_{i}\right)\right|>(\zeta \pi / 2)(i=$ $1,2, \ldots, l)$ and all critical eigenvalues that satisfy $\left|\arg \left(\chi_{i}\right)\right|=$ $(\zeta \pi / 2)(i=1,2, \ldots, l)$ own geometric multiplicity one.

\section{Existence and Uniqueness of the Solution of System (4)}

In this section, we will prove the existence and uniqueness of the solution of system (4).

Theorem 1. Let $\Lambda=\left\{\left(u_{1}, u_{2}, u_{3}\right) \in R^{3}: \max \left\{\left|u_{1}\right|,\left|u_{2}\right|,\left|u_{3}\right|\right\}\right.$ $\leq A\}$, where $A>0$ is a constant. $\forall\left(u_{10}, u_{20}, u_{30}\right) \in \Lambda$, system (4) with the initial value $\left(u_{10}, u_{20}, u_{30}\right)$ has a unique solution $U=\left(u_{1}, u_{2}, u_{3}\right) \in \Lambda$.

Proof. Define the following mapping:

$$
f(U)=\left(f_{1}(U), f_{2}(U), f_{3}(U)\right),
$$

where

$\left\{\begin{array}{l}f_{1}(U)=\beta_{1} u_{1}(t)-\kappa_{-1} u_{1}^{2}(t)-u_{1}(t) u_{2}(t)-u_{1}(t) u_{3}(t), \\ f_{2}(U)=u_{1}(t) u_{2}(t)-\beta_{5} u_{2}(t), \\ f_{3}(U)=\beta_{4} u_{3}(t)-u_{1}(t) u_{3}(t)-k_{-5} u_{3}^{2}(t) .\end{array}\right.$ 


$$
\begin{aligned}
& \|f(U)-f(\widetilde{U})\| \\
= & \mid \beta_{1} u_{1}(t)-\kappa_{-1} u_{1}^{2}(t)-u_{1}(t) u_{2}(t)-u_{1}(t) u_{3}(t) \\
& -\left[\beta_{1} \bar{u}_{1}(t)-\kappa_{-1} \bar{u}_{1}^{2}(t)-\bar{u}_{1}(t) \bar{u}_{2}(t)-\bar{u}_{1}(t) \bar{u}_{3}(t)\right] \mid \\
& +\left|u_{1}(t) u_{2}(t)-\beta_{5} u_{2}(t)-\left[\bar{u}_{1}(t) \bar{u}_{2}(t)-\beta_{5} \bar{u}_{2}(t)\right]\right| \\
& +\mid \beta_{4} u_{3}(t)-u_{1}(t) u_{3}(t)-k_{-5} u_{3}^{2}(t) \\
& -\left[\beta_{4} \bar{u}_{3}(t)-\bar{u}_{1}(t) \bar{u}_{3}(t)-k_{-5} \bar{u}_{3}^{2}(t)\right] \mid \\
\leq & \left(\beta_{1}+2 \kappa_{-1} A+A\right)\left|u_{1}(t)-\bar{u}_{1}(t)\right|+A\left|u_{2}(t)-\bar{u}_{2}(t)\right|+A\left|u_{3}(t)-\bar{u}_{3}(t)\right|+A\left|u_{1}(t)-\bar{u}_{1}(t)\right| \\
& +\left(A+\beta_{5}\right)\left|u_{2}(t)-\bar{u}_{2}(t)\right|+A\left|u_{1}(t)-\bar{u}_{1}(t)\right| \\
& +\left(\beta_{4}+A+2 \kappa_{-5} A\right)\left|u_{3}(t)-\bar{u}_{3}(t)\right| \\
= & A_{1}\left|u_{1}(t)-\bar{u}_{1}(t)\right|+A_{2}\left|u_{2}(t)-\bar{u}_{2}(t)\right|+A_{3}\left|u_{3}(t)-\bar{u}_{3}(t)\right| \\
\leq & A_{0}\|U-\bar{U}\|,
\end{aligned}
$$

where

$$
\begin{aligned}
& \left\{\begin{array}{l}
A_{1}=\beta_{1}+2 \kappa_{-1} A+3 A, \\
A_{2}=2 A+\beta_{5}, \\
A_{3}=\beta_{4}+2 A+2 \kappa_{-5} A,
\end{array}\right. \\
& A_{0}=\max \left\{A_{1}, A_{2}, A_{3}\right\} .
\end{aligned}
$$

Then $f(U)$ satisfies Lipschitz condition with respect to $U$ (see $[39,40])$. According to Banach fixed point theorem, we know that Theorem 1 is true.

\section{Suppressing Chaos via Fractional-Order PD ${ }^{\zeta}$ Controller}

In this part, we are to apply a suitable controller to eliminate the chaotic phenomenon of system (4). By virtue of the idea of Tang et al. [37], the fractional-order $\mathrm{PD}^{\zeta}$ controller can be designed as follows:

$$
\psi(t)=\varrho_{p} u_{1}(t-\theta)+\varrho_{d} \frac{\mathrm{d}^{\zeta} u_{1}(t)}{\mathrm{d} t^{\zeta}},
$$

where $\varrho_{p}$ and $\varrho_{d} \neq 1$ present the proportional control parameter and the derivative control parameter, respectively, and $\theta$ denotes a delay. Adding (13) to the first equation of system (4), we get

$$
\left\{\begin{array}{l}
\frac{\mathrm{d}^{\zeta} u_{1}(t)}{\mathrm{d} t^{\zeta}}=\beta_{1} u_{1}(t)-\kappa_{-1} u_{1}^{2}(t)-u_{1}(t) u_{2}(t)-u_{1}(t) u_{3}(t)+\psi(t) \\
\frac{\mathrm{d}^{\zeta} u_{2}(t)}{\mathrm{d} t^{\zeta}}=u_{1}(t) u_{2}(t)-\beta_{5} u_{2}(t) \\
\frac{\mathrm{d}^{\zeta} u_{3}(t)}{\mathrm{d} t^{\zeta}}=\beta_{4} u_{3}(t)-u_{1}(t) u_{3}(t)-k_{-5} u_{3}^{2}(t)
\end{array}\right.
$$

That is 


$$
\left\{\begin{array}{l}
\frac{\mathrm{d}^{\zeta} u_{1}(t)}{\mathrm{d} t^{\zeta}}=\beta_{1} u_{1}(t)-\kappa_{-1} u_{1}^{2}(t)-u_{1}(t) u_{2}(t)-u_{1}(t) u_{3}(t) \\
+\varrho_{p} u_{1}(t-\theta)+\varrho_{d} \frac{\mathrm{d}^{\zeta} u_{1}(t)}{\mathrm{d} t^{\zeta}} \\
\frac{\mathrm{d}^{\zeta} u_{2}(t)}{\mathrm{d} t^{\zeta}}=u_{1}(t) u_{2}(t)-\beta_{5} u_{2}(t) \\
\frac{\mathrm{d}^{\zeta} u_{3}(t)}{\mathrm{d} t^{\zeta}}=\beta_{4} u_{3}(t)-u_{1}(t) u_{3}(t)-k_{-5} u_{3}^{2}(t)
\end{array}\right.
$$

System (15) can be rewritten as the following form:

$$
\left\{\begin{array}{l}
\frac{\mathrm{d}^{\zeta} u_{1}(t)}{\mathrm{d} t^{\zeta}}=\frac{\beta_{1}}{1-\varrho_{d}} u_{1}(t)-\frac{\kappa_{-1}}{1-\varrho_{d}} u_{1}^{2}(t)-\frac{1}{1-\varrho_{d}} u_{1}(t) u_{2}(t) \\
-\frac{1}{1-\varrho_{d}} u_{1}(t) u_{3}(t)+\frac{\varrho_{p}}{1-\varrho_{d}} u_{1}(t-\theta), \\
\frac{\mathrm{d}^{\zeta} u_{2}(t)}{\mathrm{d} t^{\zeta}}=u_{1}(t) u_{2}(t)-\beta_{5} u_{2}(t), \\
\frac{\mathrm{d}^{\zeta} u_{3}(t)}{\mathrm{d} t^{\zeta}}=\beta_{4} u_{3}(t)-u_{1}(t) u_{3}(t)-k_{-5} u_{3}^{2}(t) .
\end{array}\right.
$$

It is not difficult to obtain that if the following condition

$$
\left(Q_{1}\right) \beta_{4}>\beta_{5},\left(\beta_{1}-\kappa_{-1}\right) \kappa_{-5}>\kappa_{4}-\kappa_{5},
$$

holds, then system (16) owns the following unique positive equilibrium $\mathcal{U}\left(u_{1}^{*}, u_{2}^{*}, u_{3}^{*}\right)$, where

$$
\left\{\begin{array}{l}
u_{1}^{*}=\beta_{5}, \\
u_{2}^{*}=\frac{\left(\beta_{1}-\kappa_{-1} \beta_{5}\right) \kappa_{-5}-\beta_{4}+\beta_{5}}{\kappa_{-5}}, \\
u_{3}^{*}=\frac{\beta_{4}-\beta_{5}}{\kappa_{-5}} .
\end{array}\right.
$$

The linear system of (16) around the positive equilibrium $\mathscr{U}\left(u_{1}^{*}, u_{2}^{*}, u_{3}^{*}\right)$ takes the following form:

$$
\left\{\begin{array}{l}
\frac{\mathrm{d}^{\zeta} u_{1}(t)}{\mathrm{d} t^{\zeta}}=a_{1} u_{1}(t)+a_{2} u_{2}(t)+a_{2} u_{3}(t)+a_{3} u_{1}(t-\theta) \\
\frac{\mathrm{d}^{\zeta} u_{2}(t)}{\mathrm{d} t^{\zeta}}=a_{4} u_{1}(t)+a_{5} u_{2}(t) \\
\frac{\mathrm{d}^{\zeta} u_{3}(t)}{\mathrm{d} t^{\zeta}}=a_{6} u_{1}(t)+a_{7} u_{3}(t)
\end{array}\right.
$$

where

$$
\left\{\begin{array}{l}
a_{1}=\frac{\beta_{1}-2 \kappa_{-1} u_{1}^{*}-u_{2}^{*}-u_{3}^{*}}{1-\varrho_{d}}, \\
a_{2}=-\frac{u_{1}^{*}}{1-\varrho_{d}}, \\
a_{3}=\frac{\varrho_{p}}{1-\varrho_{d}}, \\
a_{4}=u_{2}^{*}, \\
a_{5}=u_{1}^{*}-\beta_{5}, \\
a_{6}=-u_{3}^{*}, \\
a_{7}=\beta_{4}-u_{1}^{*}-2 \kappa_{5} u_{3}^{*} .
\end{array}\right.
$$

The characteristic equation of system (19) takes the form

$$
\operatorname{det}\left[\begin{array}{ccc}
s^{\zeta}-a_{1}-a_{3} e^{-s \theta} & -a_{2} & -a_{2} \\
-a_{4} & s^{\zeta}-a_{5} & 0 \\
-a_{6} & 0 & s^{\zeta}-a_{7}
\end{array}\right]=0 .
$$

Then,

$$
s^{3 \zeta}+b_{1} s^{2 \zeta}+b_{2} s^{\zeta}+b_{3}+\left(c_{1} s^{2 \zeta}+c_{2} s^{\zeta}+c_{3}\right) e^{-s \theta}=0,
$$

where 


$$
\left\{\begin{array}{l}
b_{1}=-\left(a_{1}+a_{5}+a_{7}\right), \\
b_{2}=a_{5} a_{7}+a_{1} a_{5}+a_{1} a_{7}-a_{2} a_{6}-a_{2} a_{4}, \\
b_{3}=a_{2} a_{5} a_{6}+a_{2} a_{4} a_{7}-a_{1} a_{5} a_{7}, \\
c_{1}=-a_{3}, \\
c_{2}=a_{3}\left(a_{5}+a_{7}\right), \\
c_{3}=-a_{3} a_{5} a_{7} .
\end{array}\right.
$$

When $\theta=0$, then (22) becomes

$$
\lambda^{3}+\left(b_{1}+c_{1}\right) \lambda^{2}+\left(b_{2}+c_{2}\right) \lambda+b_{3}+c_{3}=0 .
$$

Assuming that

$$
\left(Q_{2}\right)\left\{\begin{array}{l}
b_{1}+c_{1}>0, \\
\left(b_{1}+c_{1}\right)\left(b_{2}+c_{2}\right)>b_{3}+c_{3}, \\
\left(b_{3}+c_{3}\right)\left[\left(b_{1}+c_{1}\right)\left(b_{2}+c_{2}\right)-\left(b_{3}+c_{3}\right)\right]>0,
\end{array}\right.
$$

is true, then the three roots $\lambda_{1}, \lambda_{2}, \lambda_{3}$ of (24) satisfy $\left|\arg \left(\lambda_{1}\right)\right|>(\zeta \pi / 2),\left|\arg \left(\lambda_{2}\right)\right|>(\zeta \pi / 2)$, and $\left|\arg \left(\lambda_{3}\right)\right|>(\zeta \pi / 2)$. By virtue of Lemma 1, we can conclude that the positive equilibrium point $\mathcal{U}\left(u_{1}^{*}, u_{2}^{*}, u_{3}^{*}\right)$ of system (14) is locally asymptotically stable when $\theta=0$.

Assume that $s=i \rho=\rho(\cos (\zeta \pi / 2)+i \sin (\pi / 2))$ is the root of equation (22). It follows from (22) that

$$
\begin{aligned}
& \rho^{3 \zeta}\left(\cos \frac{3 \zeta \pi}{2}+i \sin \frac{3 \zeta \pi}{2}\right)+b_{1} \rho^{2 \zeta}(\cos \zeta \pi+i \sin \zeta \pi) \\
& \quad+b_{2} \rho^{\zeta}\left(\cos \frac{\zeta \pi}{2}+i \sin \frac{\zeta \pi}{2}\right)+b_{3} \\
& \quad+\left[c_{1} \rho^{2 \zeta}(\cos \zeta \pi+i \sin \zeta \pi)+c_{2} \rho^{\zeta}\left(\cos \frac{\zeta \pi}{2}+i \sin \frac{\zeta \pi}{2}\right)+c_{3}\right]
\end{aligned}
$$$$
\times(\cos \rho \theta-i \sin \rho \theta)=0 .
$$

Then,

$$
\left\{\begin{array}{l}
\mathscr{G}_{1} \cos \rho \theta+\mathscr{G}_{2} \sin \rho \theta=\mathscr{H}_{1}, \\
\mathscr{G}_{2} \cos \rho \theta-\mathscr{G}_{1} \sin \rho \theta=\mathscr{H}_{2},
\end{array}\right.
$$

where

$$
\left\{\begin{array}{l}
\mathscr{G}_{1}=d_{1} \rho^{2 \zeta}+d_{2} \rho^{\zeta}+d_{3}, \\
\mathscr{G}_{2}=d_{4} \rho^{2 \zeta}+d_{5} \rho^{\zeta}, \\
\mathscr{H}_{1}=e_{1} \rho^{3 \zeta}+e_{2} \rho^{2 \zeta}+e_{3} \rho^{\zeta}+e_{4}, \\
\mathscr{H}_{2}=e_{5} \rho^{3 \zeta}+e_{6} \rho^{2 \zeta}+e_{7} \rho^{\zeta},
\end{array}\right.
$$

where

$$
\left\{\begin{array}{l}
d_{1}=c_{1} \cos \zeta \pi, \\
d_{2}=c_{2} \cos \frac{\zeta \pi}{2}, \\
d_{3}=c_{3}, \\
d_{4}=c_{1} \sin \zeta \pi, \\
d_{5}=c_{2} \sin \frac{\zeta \pi}{2}, \\
e_{1}=-\cos \frac{\zeta \pi}{2}, \\
e_{2}=-b_{1} \cos \zeta \pi, \\
e_{3}=-b_{2} \cos \frac{\zeta \pi}{2}, \\
e_{4}=-b_{3}, \\
e_{5}=-\sin \frac{\zeta \pi}{2}, \\
e_{6}=-b_{1} \sin \zeta \pi, \\
e_{7}=-b_{2} \sin \frac{\zeta \pi}{2},
\end{array}\right.
$$

It follows from (27) that

$$
\begin{aligned}
\cos \rho \theta & =\frac{\mathscr{H}_{1} \mathscr{G}_{1}+\mathscr{H}_{2} \mathscr{G}_{2}}{\mathscr{G}_{1}^{2}+\mathscr{G}_{2}^{2}}, \\
\mathscr{G}_{1}^{2}+\mathscr{G}_{2}^{2} & =\mathscr{H}_{1}^{2}+\mathscr{H}_{2}^{2} .
\end{aligned}
$$

By virtue of (28) and (31), one gets

$$
\begin{aligned}
& \left(d_{1} \rho^{2 \zeta}+d_{2} \rho^{\zeta}+d_{3}\right)^{2}+\left(d_{4} \rho^{2 \zeta}+d_{5} \rho^{\zeta}\right)^{2}= \\
& \left(e_{1} \rho^{3 \zeta}+e_{2} \rho^{2 \zeta}+e_{3} \rho^{\zeta}+e_{4}\right)^{2}+\left(e_{5} \rho^{3 \zeta}+e_{6} \rho^{2 \zeta}+e_{7} \rho^{\zeta}\right)^{2},
\end{aligned}
$$

which leads to

$$
\epsilon_{1} \rho^{6 \zeta}+\epsilon_{2} \rho^{5 \zeta}+\epsilon_{3} \rho^{4 \zeta}+\epsilon_{4} \rho^{3 \zeta}+\epsilon_{5} \rho^{2 \zeta}+\epsilon_{6} \rho^{\zeta}+\epsilon_{7}=0,
$$

where 


$$
\left\{\begin{array}{l}
\epsilon_{1}=e_{1}^{2}+e_{5}^{2}, \\
\epsilon_{2}=2\left(e_{1} e_{2}+e_{5} e_{6}\right), \\
\epsilon_{3}=e_{2}^{2}+e_{6}^{2}-d_{1}^{2}-d_{4}^{2}+2\left(e_{1} e_{3}+e_{5} e_{7}\right), \\
\epsilon_{4}=2\left(e_{1} e_{4}+e_{2} e_{3}+e_{6} e_{7}-d_{1} d_{2}-d_{4} d_{5}\right), \\
\epsilon_{5}=e_{3}^{2}+e_{7}^{2}-d_{2}^{2}-d_{5}^{2}+2\left(e_{2} e_{4}-d_{1} d_{3}\right), \\
\epsilon_{6}=2\left(e_{3} e_{4}-d_{2} d_{3}\right), \\
\epsilon_{7}=e_{4}^{2}-d_{3}^{2} .
\end{array}\right.
$$

Set

$$
\Theta(\rho)=\epsilon_{1} \rho^{6 \zeta}+\epsilon_{2} \rho^{5 \zeta}+\epsilon_{3} \rho^{4 \zeta}+\epsilon_{4} \rho^{3 \zeta}+\epsilon_{5} \rho^{2 \zeta}+\epsilon_{6} \rho^{\zeta}+\epsilon_{7} .
$$

Assuming that

$$
\left(Q_{3}\right)\left|e_{4}\right|<\left|d_{3}\right|
$$

is true, since $\lim _{\rho \rightarrow \infty} \Theta(\rho)=+\infty$, then equation (33) owns at least one real positive root. So equation (22) has at least one pair of pure roots. Making use of Sun et al. [38], one can easily establish the conclusion as follows.

Lemma 2. (a) Supposing that $\epsilon_{k}>0(k=1,2,3,4,5,6)$, equation (22) owns no root with zero real parts for $\theta \geq 0$. (b) Supposing that $\left(\mathbb{Q}_{3}\right)$ holds and $\epsilon_{k}>0(k=1,2,3,4,5)$, then equation (22) owns a pair of purely imaginary roots $\pm i \rho_{0}$ if $\theta=\theta_{0}^{(l)}(l=1,2, \ldots$,$) where$

$$
\theta_{0}^{(l)}=\frac{1}{\rho_{0}}\left[\arccos \left(\frac{\mathscr{H}_{1} \mathscr{G}_{1}+\mathscr{H}_{2} \mathscr{G}_{2}}{\mathscr{G}_{1}^{2}+\mathscr{G}_{2}^{2}}\right)+2 l \pi\right]
$$

where $l=0,1, \ldots$, and $\rho_{0}>0$ represents the unique zero of $\Theta(\rho)$.

Denote $\theta_{0}=\theta_{0}^{(0)}$. Now the following hypothesis is given:

$$
\left(\mathscr{Q}_{4}\right) \mathscr{C}_{1 R} \mathscr{C}_{2 R}+\mathscr{C}_{1 I} \mathscr{C}_{2 I}>0
$$

where

$$
\left\{\begin{array}{l}
\mathscr{C}_{1 R}=3 \zeta \rho_{0}^{3 \zeta-1} \cos \frac{(3 \zeta-1) \pi}{2}+2 \zeta c_{1} \rho_{0}^{2 \zeta-1} \cos \frac{(2 \zeta-1) \pi}{2} \\
+\zeta c_{2} \rho_{0}^{\zeta-1} \cos \frac{(\zeta-1) \pi}{2}+\left[2 \zeta c_{1} \rho_{0}^{2 \zeta-1} \cos \frac{(2 \zeta-1) \pi}{2}+\zeta c_{2} \rho_{0}^{\zeta-1} \cos \frac{(\zeta-1) \pi}{2}\right] \cos \rho_{0} \theta_{0}+\sin \rho_{0} \theta_{0} \\
\times\left[2 \zeta c_{1} \rho_{0}^{2 \zeta-1} \sin \frac{(2 \zeta-1) \pi}{2}+\zeta c_{2} \rho_{0}^{\zeta-1} \sin \frac{(\zeta-1) \pi}{2}\right] \\
\mathscr{C}_{1 I}=3 \zeta \rho_{0}^{3 \zeta-1} \sin \frac{(3 \zeta-1) \pi}{2}+2 \zeta c_{1} \rho_{0}^{2 \zeta-1} \sin \frac{(2 \zeta-1) \pi}{2} \\
+\zeta c_{2} \rho_{0}^{\zeta-1} \sin \frac{(\zeta-1) \pi}{2}-\left[2 \zeta c_{1} \rho_{0}^{2 \zeta-1} \cos \frac{(2 \zeta-1) \pi}{2}+\zeta c_{2} \rho_{0}^{\zeta-1} \cos \frac{(\zeta-1) \pi}{2}\right] \sin \rho_{0} \theta_{0}+\cos \rho_{0} \theta_{0} \\
\times\left[2 \zeta c_{1} \rho_{0}^{2 \zeta-1} \sin \frac{(2 \zeta-1) \pi}{2}+\zeta c_{2} \rho_{0}^{\zeta-1} \sin \frac{(\zeta-1) \pi}{2}\right] \\
+\left(c_{1} \rho_{0}^{2 \zeta} \sin \zeta \pi+c_{2} \rho_{0}^{\zeta} \sin \frac{\zeta \pi}{2}+c_{3}\right) \rho_{0} \sin \rho_{0} \theta_{0} . \\
\mathscr{C}_{2 R}=\left(c_{1} \rho_{0}^{2 \zeta} \cos \zeta \pi+c_{2} \rho_{0}^{\zeta} \cos \frac{\zeta \pi}{2}+c_{3}\right) \rho_{0} \sin \rho_{0} \theta_{0} \\
-\left(c_{1} \rho_{0}^{2 \zeta} \sin \zeta \pi+c_{2} \rho_{0}^{\zeta} \sin \frac{\zeta \pi}{2}+c_{3}\right) \rho_{0} \cos \rho_{0} \theta_{0} \\
\mathscr{C}_{2 I}=\left(c_{1} \rho_{0}^{2 \zeta} \cos \zeta \pi+c_{2} \rho_{0}^{\zeta} \cos \frac{\zeta \pi}{2}+c_{3}\right) \rho_{0} \cos \rho_{0} \theta_{0} \\
\\
\\
\end{array}\right.
$$


Lemma 3. Let $s(\theta)=\phi_{1}(\theta)+i \phi_{2}(\theta)$ be the root of (22) at $\theta=\theta_{0}$ satisfying $\phi_{1}\left(\theta_{0}\right)=0, \phi_{2}\left(\theta_{0}\right)=\rho_{0}$, then $\operatorname{Re}(d s /$ $d \theta)\left.\right|_{\theta=\theta_{0}, \rho=\rho_{0}}>0$.

Proof. Making use of (22), we get

$$
\begin{aligned}
& \left(3 \zeta s^{3 \zeta-1}+2 \zeta b_{1} s^{2 \zeta-1}+\zeta b_{2} s^{\zeta-1}\right) \frac{\mathrm{d} s}{\mathrm{~d} \theta} \\
& +\left(2 \zeta c_{1} s^{2 \zeta-1}+\zeta c_{2} s^{\zeta-1}\right) e^{-s \theta} \frac{\mathrm{d} s}{\mathrm{~d} \theta} \\
& -e^{-s \theta}\left(\frac{\mathrm{d} s}{\mathrm{~d} \theta} \theta+s\right)\left(c_{1} s^{2 \zeta}+c_{2} s^{\zeta}+c_{3}\right)=0,
\end{aligned}
$$

which leads to

$$
\begin{aligned}
& {\left[3 \zeta s^{3 \zeta-1}+2 \zeta b_{1} s^{2 \zeta-1}+\zeta b_{2} s^{\zeta-1}+\left(2 \zeta c_{1} s^{2 \zeta-1}+\zeta c_{2} s^{\zeta-1}\right) e^{-s \theta}-\theta e^{-s \theta}\left(c_{1} s^{2 \zeta}+c_{2} s^{\zeta}+c_{3}\right)\right] \frac{\mathrm{d} s}{\mathrm{~d} \theta} } \\
= & s e^{-s \theta}\left(c_{1} s^{2 \zeta}+c_{2} s^{\zeta}+c_{3}\right) .
\end{aligned}
$$

Then,

$$
\left(\frac{\mathrm{d} s}{\mathrm{~d} \theta}\right)^{-1}=\frac{\mathscr{C}_{1}(s)}{\mathscr{C}_{2}(s)}-\frac{\theta}{s},
$$

where

$$
\left\{\begin{array}{l}
\mathscr{C}_{1}(s)=3 \zeta s^{3 \zeta-1}+2 \zeta b_{1} s^{2 \zeta-1}+\zeta b_{2} s^{\zeta-1} \\
+\left(2 \zeta c_{1} s^{2 \zeta-1}+\zeta c_{2} s^{\zeta-1}\right) e^{-s \theta} \\
\mathscr{C}_{2}(s)=s e^{-s \theta}\left(c_{1} s^{2 \zeta}+c_{2} s^{\zeta}+c_{3}\right) .
\end{array}\right.
$$

Then,

$$
\begin{aligned}
\operatorname{Re}\left[\left(\frac{\mathrm{d} s}{\mathrm{~d} \theta}\right)^{-1}\right]_{\theta=\theta_{0}, \rho=\rho_{0}} & =\operatorname{Re}\left[\frac{\mathscr{C}_{1}(s)}{\mathscr{C}_{2}(s)}\right]_{\theta=\theta_{0}, \rho=\rho_{0}} \\
& =\frac{\mathscr{C}_{1 R} \mathscr{C}_{2 R}+\mathscr{C}_{1 I} \mathscr{C}_{2 I}}{\mathscr{C}_{2 R}^{2}+\mathscr{C}_{2 I}^{2}} .
\end{aligned}
$$

In view of $\left(\mathscr{Q}_{4}\right)$, we have

$$
\operatorname{Re}\left[\left(\frac{\mathrm{d} s}{\mathrm{~d} \theta}\right)^{-1}\right]_{\theta=\theta_{0}, \rho=\rho_{0}}>0
$$

which completes the proof.

Making use of Lemma 1, we can easily obtain the following conclusion.

Theorem 2. Supposing that $\left(\mathbb{Q}_{1}\right)-\left(\mathbb{Q}_{4}\right)$ hold, then the positive equilibrium point $\mathcal{U}\left(u_{1}^{*}, u_{2}^{*}, u_{3}^{*}\right)$ of system (16) is locally asymptotically stable if the time delay $\theta$ lies in the interval $\left[0, \theta_{0}\right)$ and the Hopf bifurcation phenomenon of system (16) will arise near the positive equilibrium point $\mathcal{U}\left(u_{1}^{*}, u_{2}^{*}, u_{3}^{*}\right)$ if $\theta=\theta_{0}$.

Remark 1. Xu and $\mathrm{Wu}$ [17] dealt with the chaos control for an integer-order chaotic chemical reaction model by timedelay feedback control technique. This manuscript deals with the chaos control issue for a fractional-order chaotic chemical reaction model via a fractional-order $\mathrm{PD}^{\zeta}$ controller. The model and the research approach is very different from those in [17]. From this viewpoint, we think that the obtained results and the research method of this manuscript supplement the work of [17] and promote the development of the chaos control theory of fractional-order differential equation to some degree.

Remark 2. In this paper, we use the fractional-order $\mathrm{PD}^{\zeta}$ controller to control the chaos of the fractional-order chaotic chemical reaction model (4). Compared with the time delay feedback controller, the fractional-order $\mathrm{PD}^{\zeta}$ controller has more adjustable parameters and then can control the chaos of model (4) neatly.

\section{Example}

Consider the following controlled fractional-order chaotic chemical reaction model: 

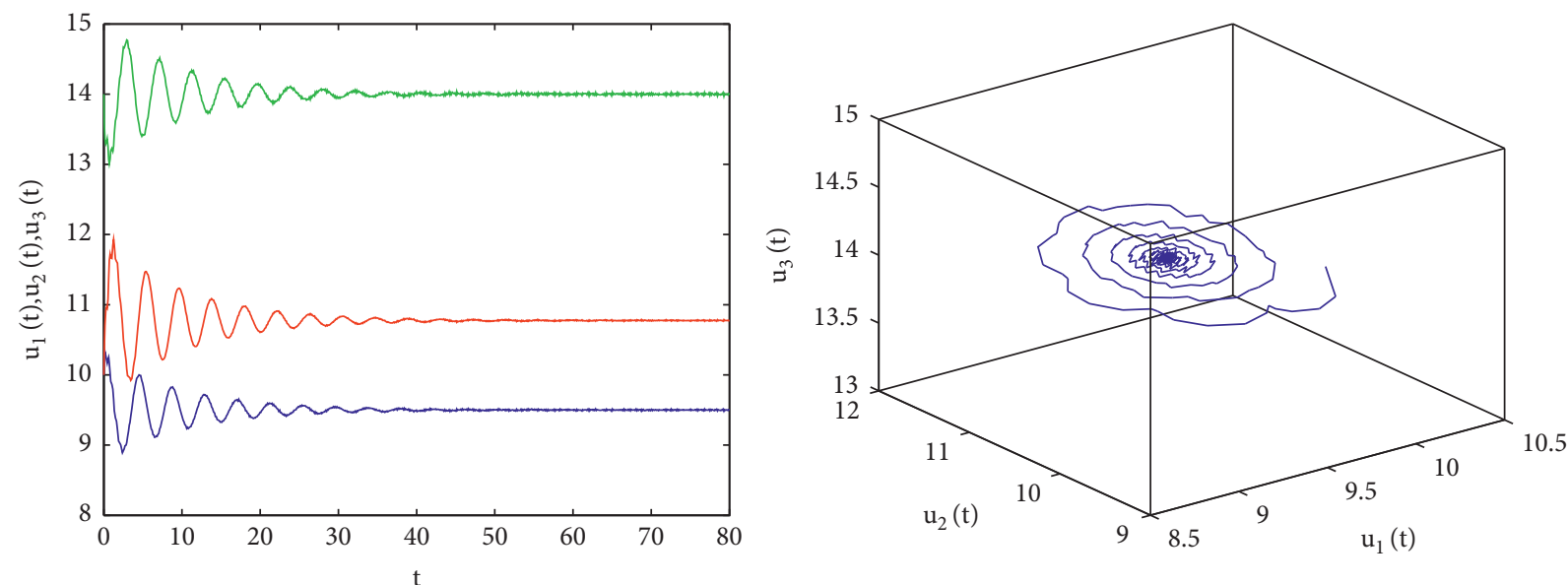

Figure 2: Computer simulation figures of the controlled fractional-order chaotic chemical reaction model $(46)$ with $\theta=0.20<\theta_{0}=0.25$. The blue line represents $u_{1}(t)$, the red line represents $u_{2}(t)$, and the green line represents $u_{3}(t)$.
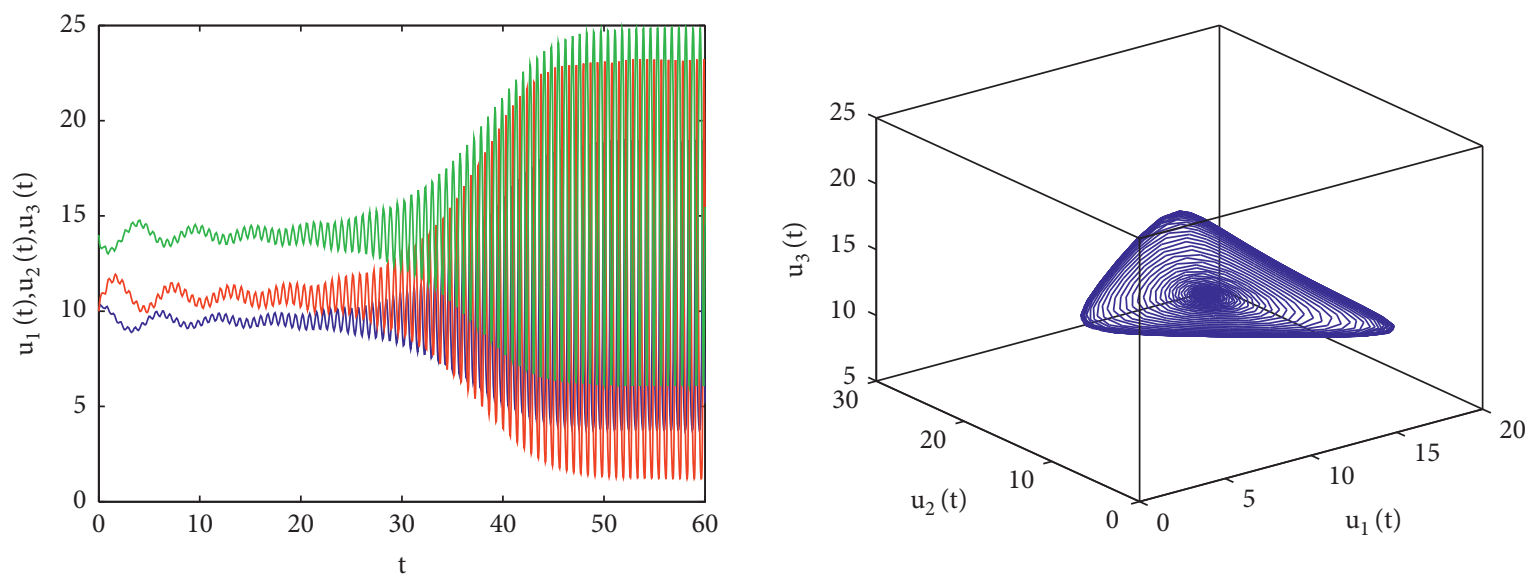

FiguRE 3: Computer simulation figures of the controlled fractional-order chaotic chemical reaction model $(46)$ with $\theta=0.28>\theta_{0}=0.25$. The blue line represents $u_{1}(t)$, the red line represents $u_{2}(t)$, and the green line represents $u_{3}(t)$.

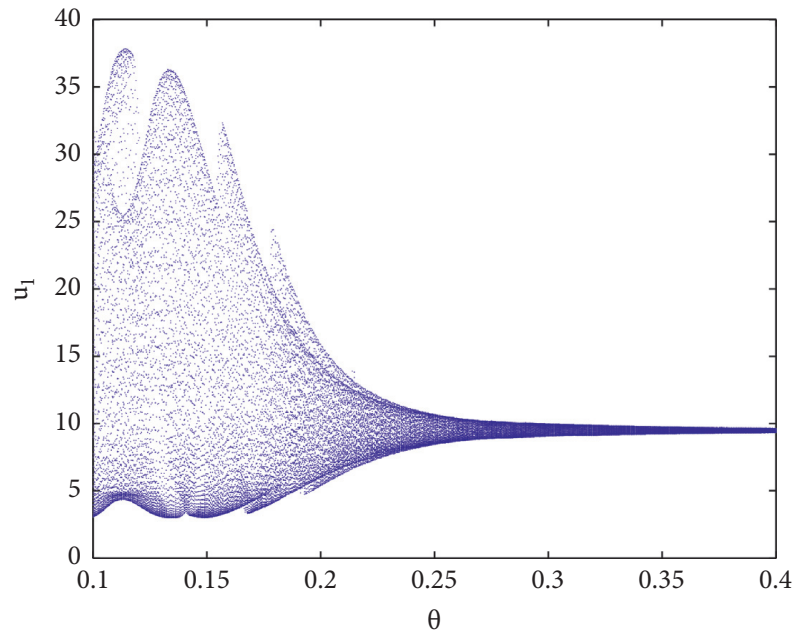

Figure 4: Bifurcation plot of the controlled fractional-order chaotic chemical reaction model (46): $\theta-u_{1}$. 
$$
\left\{\begin{array}{l}
\frac{\mathrm{d}^{\zeta} u_{1}(t)}{\mathrm{d} t^{\zeta}}=\beta_{1} u_{1}(t)-\kappa_{-1} u_{1}^{2}(t)-u_{1}(t) u_{2}(t)-u_{1}(t) u_{3}(t) \\
+\varrho_{p} u_{1}(t-\theta)+\varrho_{d} \frac{\mathrm{d}^{\zeta} u_{1}(t)}{\mathrm{d} t^{\zeta}}, \\
\frac{\mathrm{d}^{\zeta} u_{2}(t)}{\mathrm{d} t^{\zeta}}=u_{1}(t) u_{2}(t)-\beta_{5} u_{2}(t), \\
\frac{\mathrm{d}^{\zeta} u_{3}(t)}{\mathrm{d} t^{\zeta}}=\beta_{4} u_{3}(t)-u_{1}(t) u_{3}(t)-k_{-5} u_{3}^{2}(t),
\end{array}\right.
$$

where $\zeta=0.97, \beta_{1}=30, \kappa_{-1}=0.55, \beta_{5}=9.5, \beta_{4}=16.5, \kappa_{-5}=0.5$. Let $\varrho_{p}=0.5, \varrho_{d}=0.9$. It is not difficult to obtain the unique positive equilibrium point of system $(46)$ is $\mathcal{U}(9.5,10.775,14)$. By direct computation via MATLAB software, we can easily get $\rho_{0}=4.0239$ and $\theta_{0}=0.25$. The three assumptions $\left(Q_{1}\right)-\left(Q_{4}\right)$ of Theorem 2 are easily verified to be right. So we can conclude that the positive equilibrium point $\mathcal{U}(9.5$, $10.775,14)$ of system (46) is locally asymptotically stable if the time delay $\theta$ lies in the interval $[0,0.25)$ and the Hopf bifurcation phenomenon for system (46) will arise near the positive equilibrium point $\mathcal{U}(9.5,10.775,14)$ if $\theta=0.25$. In this paper, we use the predictor-correctors approach $[39,41,42]$ to discretize system (46) and by virtue of the MATLAB software to carry out numerical simulations. In order to display these results, we select two sets of different delay parameters. Firstly, we choose $\theta=0.20<\theta_{0}=0.25$, and the software simulation plots are presented in Figure 2, which implies that $u_{1} \longrightarrow 9.5, u_{2} \longrightarrow 10.775, u_{3} \longrightarrow 14$ as the time $t$ tends to infinity. From the chemical point of view, the mole fraction of the constituent $\mathscr{X}$ will be close to 9.5 , the mole fraction of the constituent $\mathscr{Y}$ will be close to 10.775 , and the mole fraction of the constituent $\mathscr{Z}$ will be close to 14 . Secondly, we choose $\theta=0.28>\theta_{0}=0.25$, and the software simulation plots are presented in Figure 3, which implies that a Hopf bifurcation periodic solution of system (46) will arise near the positive equilibrium point $\mathcal{U}(9.5,10.775,14)$ as the time $t$ tends to infinity. From the chemical point of view, the mole fraction of the constituent $\mathscr{X}$, the mole fraction of the constituent $\mathcal{Y}$, and the mole fraction of the constituent $\mathscr{Z}$ will remain periodically oscillatory situations near the values $9.5,10.775,14$, respectively. Furthermore, we give the bifurcation plot, which can be seen in Figure 4, to indicate that the bifurcation value of system (46) is 0.25 .

\section{Conclusions}

Suppressing the chaotic behavior of nonlinear dynamical systems has been a significant and classic issue in many disciplines. For a long time, the suppression of chaos has attracted much attention from many scholars in mathematics, physics, chemistry, engineering, and numerous other areas. In the present manuscript, based on the earlier publications, we set up a novel fractional-order chaotic chemical reaction model. Taking advantage of an appropriate fractional-order $\mathrm{PD}^{\zeta}$ controller, we can effectively eliminate the chaotic phenomenon of the involved fractional-order chaotic chemical reaction model. A delay-independent sufficient condition to guarantee the stability and the creation of Hopf bifurcation of the fractional-order controlled chaotic chemical reaction model is built. The exploration manifests that the delay occurring in fractionalorder $\mathrm{PD}^{\zeta}$ controller is the key factor in suppressing the chaotic phenomenon of the fractional-order chaotic chemical reaction model. The derived conclusions of this manuscript are entirely new and the exploration approach of this manuscript can also be utilized to inquire into numerous chaos control problem of lots of fractional-order chaotic dynamical systems.

\section{Data Availability}

No data were used to support this study.

\section{Conflicts of Interest}

The authors declare that they have no conflicts of interest.

\section{References}

[1] C. T. Dhanya and D. Nagesh Kumar, "Multivariate nonlinear ensemble prediction of daily chaotic rainfall with climate inputs," Journal of Hydrology, vol. 403, no. 3-4, pp. 292-306, 2011.

[2] S. Kumar, R. Kumar, C. Cattani, and B. Samet, "Chaotic behaviour of fractional predator-prey dynamical system," Chaos, Solitons \& Fractals, vol. 135, Article ID 109811, 2020.

[3] K. P. Harikrishnan, R. Misra, and G. Ambika, "Quantifying information loss on chaotic attractors through recurrence networks," Physics Letters A, vol. 383, no. 27, Article ID 125854, 2019.

[4] A. M. Wojtusiak, A. G. Balanov, and S. E. Savel'ev, "Intermittent and metastable chaos in a memristive artificial neuron with inertia," Chaos, Solitons \& Fractals, vol. 142, Article ID 110383, 2021.

[5] I. Ahmad, A. Ouannas, M. Shafiq, V.-T. Pham, and D. Baleanu, "Finite-time stabilization of a perturbed chaotic finance model," Journal of Advanced Research, vol. 32, pp. 1-14, 2021.

[6] S. Harshavarthini, R. Sakthivel, Y.-K. Ma, and M. Muslim, "Finite-time resilient fault-tolerant investment policy scheme for chaotic nonlinear finance system," Chaos, Solitons \& Fractals, vol. 132, Article ID 109567, 2020.

[7] G. Chen, "A simple adaptive feedback control method for chaos and hyper-chaos control," Applied Mathematics and Computation, vol. 217, no. 17, pp. 7258-7264, 2011.

[8] J. Du, T. Huang, Z. Sheng, and H. Zhang, "A new method to control chaos in an economic system," Applied Mathematics and Computation, vol. 217, no. 6, pp. 2370-2380, 2010.

[9] E. Ott, C. Grebogi, and J. A. Yorke, "Controlling chaos," Physical Review Letters, vol. 64, no. 11, pp. 1196-1199, 1990.

[10] A. S. de Paula and M. A. Savi, "Controlling chaos in a nonlinear pendulum using an extended time-delayed feedback control method," Chaos, Solitons \& Fractals, vol. 42, no. 5, pp. 2981-2988, 2009.

[11] H. Sadeghian, H. Salarieh, A. Alasty, and A. Meghdari, "On the control of chaos via fractional delayed feedback method," 
Computers \& Mathematics with Applications, vol. 62, no. 3, pp. 1482-1491, 2011.

[12] Y. Han, J. Ding, L. Du, and Y. Lei, "Control and anti-control of chaos based on the moving largest Lyapunov exponent using reinforcement learning," Physica D: Nonlinear Phenomena, vol. 428, Article ID 133068, 2021.

[13] X. Yin, J. She, Z. Liu, M. Wu, and O. Kaynak, "Chaos suppression in speed control for permanent-magnet-synchronous-motor drive system," Journal of the Franklin Institute, vol. 357, no. 18, pp. 13283-13303, 2020.

[14] X. Chen and J. Zhou, "The complexity analysis and chaos control in omni-channel supply chain with consumer migration and advertising cost sharing," Chaos, Solitons \& Fractals, vol. 146, Article ID 110884, 2021.

[15] P. Geysermans and F. Baras, "Particle simulation of chemical chaos," The Journal of Chemical Physics, vol. 105, no. 4, pp. 1402-1408, 1996.

[16] P. Geysermans and G. Nicolis, "Thermodynamic fluctuations and chemical chaos in a well-stirred reactor: a master equation analysis," The Journal of Chemical Physics, vol. 99, no. 11, pp. 8964-8969, 1993.

[17] C. Xu and Y. Wu, "Bifurcation and control of chaos in a chemical system," Applied Mathematical Modelling, vol. 39, no. 8, pp. 2295-2310, 2015.

[18] C. J. Xu, W. Zhang, C. Aouiti, Z. X. Liu, M. X. Liao, and P. L. Li, "Further investigation on bifurcation and their control of fractional-order BAM neural networks involving four neurons and multiple delays," Mathematical Methods in the Applied Sciences, 2021.

[19] C. Xu, Z. Liu, L. Yao, and C. Aouiti, "Further exploration on bifurcation of fractional-order six-neuron bi-directional associative memory neural networks with multi-delays," Applied Mathematics and Computation, vol. 410, Article ID 126458, 2021.

[20] F. B. Yousef, A. Yousef, and C. Maji, "Effects of fear in a fractional-order predator-prey system with predator densitydependent prey mortality," Chaos, Solitons \& Fractals, vol. 145, Article ID 110711, 2021.

[21] C. Huang, H. Liu, X. Chen et al., "Dynamic optimal control of enhancing feedback treatment for a delayed fractional order predator-prey model," Physica A: Statistical Mechanics and its Applications, vol. 554, Article ID 124136, 2020.

[22] C. Xu, M. Liao, P. Li, Y. Guo, and Z. Liu, "Bifurcation properties for fractional order delayed BAM neural networks," Cognitive Computation, vol. 13, no. 2, pp. 322-356, 2021.

[23] F. A. Rihan and C. Rajivganthi, "Dynamics of fractional-order delay differential model of prey-predator system with Holling-type III and infection among predators," Chaos, Solitons \& Fractals, vol. 141, Article ID 110365, 2020.

[24] X. Nie, P. Liu, J. Liang, and J. Cao, "Exact coexistence and locally asymptotic stability of multiple equilibria for fractionalorder delayed Hopfield neural networks with Gaussian activation function," Neural Networks, vol. 142, pp. 690-700, 2021.

[25] F. Zhang, T. Huang, Q. Wu, and Z. Zeng, "Multistability of delayed fractional-order competitive neural networks," Neural Networks, vol. 140, pp. 325-335, 2021.

[26] C. Xu, Z. Liu, M. Liao, P. Li, Q. Xiao, and S. Yuan, "Fractionalorder bidirectional associate memory (BAM) neural networks with multiple delays: the case of Hopf bifurcation," Mathematics and Computers in Simulation, vol. 182, pp. 471-494, 2021.

[27] L. Ke, "Mittag-Leffler stability and asymptotic $\omega$-periodicity of fractional-order inertial neural networks with time-delays," Neurocomputing, vol. 465, pp. 53-62, 2021.
[28] F. F. Du and J. G. Lu, Finite-Time Stability of Fractional-Order Fuzzy Cellular Neural Networks with Time Delays, Fuzzy Sets and Systems, 2021.

[29] M. Xiao, W. X. Zheng, J. Lin, G. Jiang, L. Zhao, and J. Cao, "Fractional-order PD control at Hopf bifurcations in delayed fractional-order small-world networks," Journal of the Franklin Institute, vol. 354, no. 17, pp. 7643-7667, 2017.

[30] C. Huang, H. Liu, X. Shi et al., "Bifurcations in a fractionalorder neural network with multiple leakage delays," Neural Networks, vol. 131, pp. 115-126, 2020.

[31] J. Alidousti and E. Ghafari, "Dynamic behavior of a fractional order prey-predator model with group defense," Chaos, Solitons \& Fractals, vol. 134, Article ID 109688, 2020.

[32] W. Wang and M. A. Khan, "Analysis and numerical simulation of fractional model of bank data with fractal-fractional Atangana-Baleanu derivative," Journal of Computational and Applied Mathematics, vol. 369, Article ID 112646, 2020.

[33] C. Xu, M. Liao, P. Li, and S. Yuan, "Impact of leakage delay on bifurcation in fractional-order complex-valued neural networks," Chaos, Solitons \& Fractals, vol. 142, Article ID 110535, 2021.

[34] C. Xu, W. Zhang, W. Zhang et al., "Bifurcation dynamics in a fractional-order Oregonator model including time delay," MATCH Communications in Mathematical and in Computer Chemistry, vol. 87, no. 2, pp. 397-414, 2022.

[35] I. Podlubny, Fractional Differential Equations, Academic Press, New York, NY, USA, 1999.

[36] D. Matignon, "Stability results for fractional differential equations with applications to control processing, Computational engineering in systems and application multi-conference, IMACS," IEEE-SMC Proceedings, Lille, vol. 2, pp. 963-968, 1996.

[37] Y. Tang, M. Xiao, G. Jiang, J. Lin, J. Cao, and W. X. Zheng, "Fractional-order PD control at Hopf bifurcations in a fractional-order congestion control system," Nonlinear Dynamics, vol. 90, no. 3, pp. 2185-2198, 2017.

[38] Q. Sun, M. Xiao, and B. Tao, "Local bifurcation analysis of a fractional-order dynamic model of genetic regulatory networks with delays," Neural Processing Letters, vol. 47, no. 3, pp. 1285-1296, 2018.

[39] A. M. Sayed, A. E. Matouk, S. Kumar, V. Ali, and L. Bachioua, "Chaotic dynamics and chaos control in a fractional-order satellite model and its time-delay counterpart," Discrete Dynamics in Nature and Society, vol. 2021, Article ID 5542908, 11 pages, 2021.

[40] H.-L. Li, L. Zhang, C. Hu, Y.-L. Jiang, and Z. Teng, "Dynamical analysis of a fractional-order predator-prey model incorporating a prey refuge," Journal of Applied Mathematics and Computing, vol. 54, no. 1-2, pp. 435-449, 2017.

[41] K. Diethelm, "An algorithm for the numerical solution of differential equations of fractional order," Electronic Transactions on Numerical Analysis, vol. 5, pp. 1-6, 1997.

[42] K. Diethelm, N. J. Ford, and A. D. Freed, "A predictor-corrector approach for the numerical solution of fractional differential equations," Nonlinear Dynamics, vol. 29, no. 1/4, pp. 3-22, 2002. 